Movement-Related Feedback and Temporal Accuracy in Clarinet Performance Author(s): Caroline Palmer, Erik Koopmans, Janeen D. Loehr, Christine Carter Reviewed work(s):

Source: Music Perception: An Interdisciplinary Journal, Vol. 26, No. 5 (June 2009), pp. 439-449 Published by: University of California Press

Stable URL: http://www.jstor.org/stable/10.1525/mp.2009.26.5.439

Accessed: 09/07/2012 09:04

Your use of the JSTOR archive indicates your acceptance of the Terms \& Conditions of Use, available at http://www.jstor.org/page/info/about/policies/terms.jsp

JSTOR is a not-for-profit service that helps scholars, researchers, and students discover, use, and build upon a wide range of content in a trusted digital archive. We use information technology and tools to increase productivity and facilitate new forms of scholarship. For more information about JSTOR, please contact support@jstor.org. 


\section{Movement-Related Feedback and Temporal Accuracy in Clarinet Performance}

\author{
Caroline Palmer, Erik Koopmans, \\ Janeen D. Loehr, and Christine Carter \\ McGill University, Montreal, Canada
}

SENSORY INFORMATION AVAILABLE WHEN MUSICIANS' fingers arrive on instrument keys contributes to temporal accuracy in piano performance (Goebl \& Palmer, 2008). The hypothesis that timing accuracy is related to sensory (tactile) information available at finger-key contact was extended to clarinetists' finger movements during key depressions and releases that, together with breathing, determine the timing of tone onsets. Skilled clarinetists performed melodies at different tempi in a synchronization task while their movements were recorded with motion capture. Finger accelerations indicated consistent kinematic landmarks when fingers made initial contact with or release from the key surface. Performances that contained more kinematic landmarks had reduced timing error. The magnitude of finger accelerations on key contact and release was positively correlated with increased temporal accuracy during the subsequent keystroke. These findings suggest that sensory information available at finger-key contact enhances the temporal accuracy of music performance.

Received August 29, 2008, accepted February 19, 2009.

Key words: synchronization, timing accuracy, wind performance, motion capture, sensory feedback

$\mathrm{W}$ HAT ROLE DOES TACTILE AND proprioceptive feedback play in the production of fast finger sequences typical of music performance? Instruments such as piano, strings, and wind instruments require fine control of finger movements in order to generate performances with high temporal precision and accuracy. The arrival of fingers on instrumental surfaces can provide important sensory information to guide performance. The precision of finger movements is attributed in part to the receptors distributed in the skin of the fingertips; these tactile sensory inputs permit high sensitivity and subsequent response to changes in force loads (Flanagan \& Johansson, 2002). Tactile feedback may be more salient than proprioceptive sensations, due to the high density of mechanoreceptor innervation in the finger-tip skin and the large cortical areas involved in processing tactile information from the fingers (Jones, 1996).

Although there are few studies of sensory feedback from finger-key contact in music performance, several studies of sensory feedback during tapping tasks indicate that tactile information plays an important role in the control of timing. Aschersleben and colleagues proposed the Sensory Accumulator Model (SAM) to explain how people synchronize finger tapping with an auditory pacing sequence, and how this synchronization is influenced by tactile information (Aschersleben, Gehrke, \& Prinz, 2001). When participants tap in synchrony with an auditory pacing sequence, their taps precede the pacing sequence, termed a negative asynchrony (Aschersleben, 2002). According to SAM, the brain registers the time of a tap when afferent signals associated with the tap accumulate past a certain threshold, which is compared with the time at which the brain receives information from the metronome's onset. Based on the assumption that tactile information from the finger accumulates more slowly than auditory information from the metronome, participants must tap before the metronome's onset to maintain subjective synchrony, leading to a negative asynchrony. A similar prediction arises from the earlier nerve conduction hypothesis (Fraisse, 1980), based on the fact that finger-brain connections take longer than ear-brain connections.

The sensory accumulator model makes several additional predictions, based on the assumption that central processing delays are changed by the accumulation functions of sensory signals. Production with the same effector can lead to larger or smaller negative asynchronies, depending on factors that influence the rate of accumulation. Rapid tactile input should increase the speed of processing and enhance synchronization accuracy. For example, producing a finger tap with more force should lead to faster accumulation of tactile information and correspondingly reduced negative asynchronies (Aschersleben, 2002). This prediction is supported by findings that tapping with larger movement amplitudes resulted in greater force at surface contact, more afferent information, and smaller

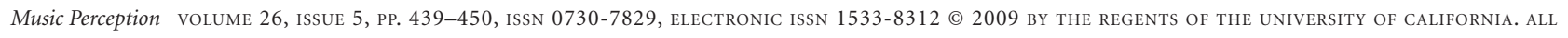
RIGHTS RESERVED. PLEASE DIRECT ALL REQUESTS FOR PERMISSION TO PHOTOCOPY OR REPRODUCE ARTICLE CONTENT THROUGH THE UNIVERSITY OF CALIFORNIA PRESS'S RIGHTS AND PERMISSIONS WEBSITE, HTTP://WWW.UCPRESSJOURNALS.COM/REPRINTINFO.ASP. DOI:10.1525/MP.2009.26.5.439 
asynchronies than tapping with smaller finger amplitudes (Aschersleben, 2002; Aschersleben, Gehrke, \& Prinz, 2004). Likewise, when afferent tactile feedback is blocked through local anesthesia, negative mean asynchronies increase due to the reduced number of feedback signals from mechanoreceptors that co-occur upon impact (Aschersleben et al., 2001). We investigated the role of tactile information available to clarinetists in the timing of finger sequences in music performance. Kinematic measures, including the peak accelerations that occur when a finger contacts a piano key or a clarinet key, often are used as indirect measures of tactile information (Aschersleben et al., 2001; Gentilucci, Toni, Daprati \& Gangitano, 1997; Goebl \& Palmer, 2008; Gordon \& Soechting, 1995). Mechanoreceptors in the fingertips are sensitive to indentation of the fingertip; the rate at which these receptors discharge increases with the force of the finger indentation (Knibestöl \& Valbo, 1980; Werner \& Mountcastle, 1965). Peak acceleration is directly related to the amount of force applied at the fingertip and therefore indicates the amount of tactile information available at the fingertip. Receptors in the tendons that connect muscle to bone also encode the amount of force produced by contracting muscles (Macefield, 2005). However, anesthetizing the fingertips (which removes tactile feedback but does not affect the force receptors) has a similar impact on the negative asynchrony as that of movement amplitude (Aschersleben et al., 2001). This suggests that tactile information may be the primary source of movementrelated feedback.

Finger accelerations have been implicated in the role of tactile information in musicians' timing accuracy. Goebl and Palmer (2008) measured individual differences related to touch in pianists' finger accelerations as the fingers first contacted the piano keys (called kinematic landmarks). Some pianists' finger movements showed persistently high proportions of these landmarks across all performances, whereas other pianists' performances exhibited high proportions only at faster tempi, when fingers were raised higher. Most important, these individual differences were related to performers' timing accuracy: Performances that contained more peak accelerations as fingers contacted piano keys showed increased temporal accuracy in the upcoming keystroke. Pianists also tend to raise their fingers higher at faster tempi (Dalla Bella \& Palmer, 2004; Loehr \& Palmer, 2007), perhaps seeking additional afferent information as suggested by Aschersleben (2002). Clarinetists also raise their fingers higher at faster tempi (Palmer, Carter, Koopmans, \& Loehr, 2007), despite the fact that finger movements change only the timing (and not the intensity or loudness) of sounded clarinet tones. Thus, the manipulation of finger height and acceleration at key contact in clarinet performance, as well as piano performance, may be an attempt to gain additional sensory information. Several variables cooccur in piano performance when fingers strike keys; the speed with which fingers depress keys is tied directly to pitch production and tone intensity in piano performance, and pianists' finger-key contacts may be related to those properties of sound production. Wind instruments in contrast rely on breathing for tone production and intensity, and on key depressions and releases for pitch change. Thus, wind instruments such as clarinet offer specific grounds for teasing apart the sound-related and movement-related (tactile) feedback offered by finger-key contacts, and the relationship with temporal accuracy.

Clarinet keys themselves have some important similarities and differences from keys on other instruments that may influence the sensory feedback available to performers. Similar to piano keys, clarinet keys have springs that provide resistance to fingers when they are depressed from their rest position. Thus, kinematic landmarks in finger motion may be expected when keys leave or return to their resting state (when spring resistance changes). In contrast to piano keys, which operate independently, some clarinet keys (including keys operated by the right hand's index, middle, and ring fingers under study here) are linked in their motion by a bar that depresses the springs associated with all keys as soon as the first key is depressed; the fingers must still move independently to cover the holes (thus altering the pitch), but the springs that provide resistance are already depressed. Therefore, the tactile information available to clarinetists from these keys will depend on the order of key depressions; the first key depressed and last key released will receive more change in spring resistance than other keys, and the kinematic landmarks may change as a result. We document here the finger motion dynamics associated with keypresses and key releases in clarinet performance, and whether motion differences are associated with the temporal accuracy of performance.

We investigated clarinetists' finger movements as they performed simple melodies at different tempi, and examined how their finger-surface contact accelerations corresponded with temporal accuracy. Two types of kinematic landmarks were examined that result from interaction of clarinetists' fingers with the clarinet keys: finger-key (FK) contact and key-bottom (KB) contact. An example of finger-key contact in clarinet performance is shown in Figure 1. The top panel shows the index finger hovering above the clarinet key; the middle picture shows 

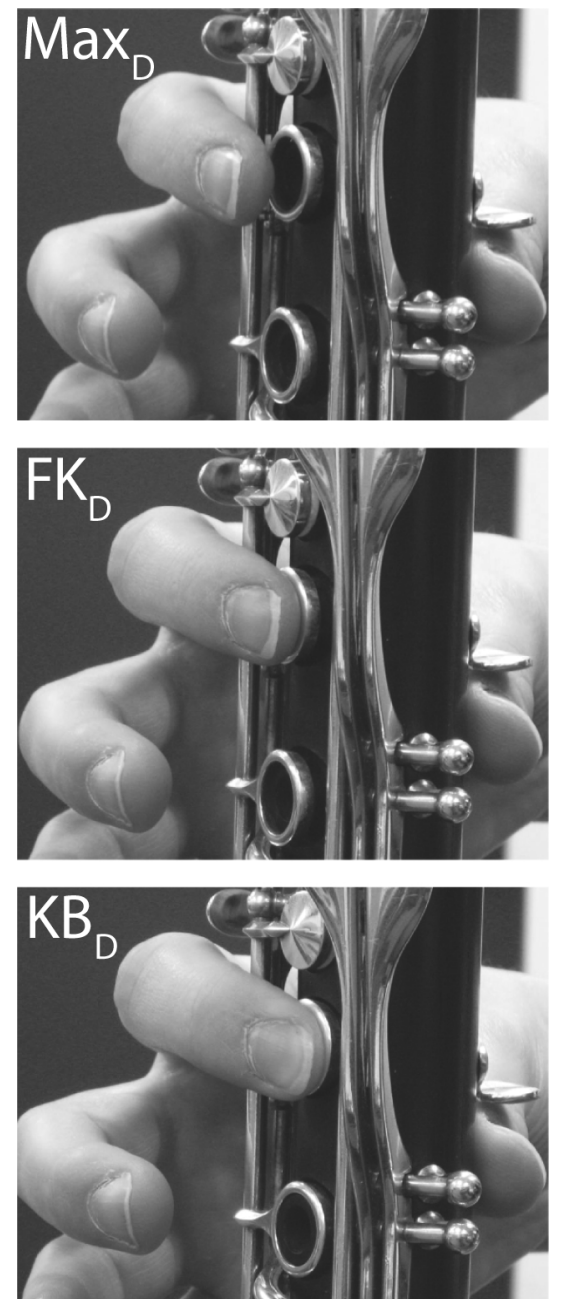

FIGURE 1. Photograph of finger position relative to clarinet keys prior to key depression. Top picture shows finger at maximum height above key $\left(M A X_{D}\right)$, middle picture shows initial finger contact with key surface $\left(F K_{D}\right)$, and bottom picture shows finger after key is depressed to bottom (KB).

the index finger when it first arrives on the key (FK), and the bottom picture shows the key reaching bottom $(\mathrm{KB})$. Although the index finger was the primary focus of this study, the same FK and KB landmarks can be documented for other finger-key contacts in clarinet performance. Key-bottom contacts occur when the finger is stopped as the clarinet key arrives at the keybed during depression, called $\mathrm{KB}_{\mathrm{D}}$, and when the finger begins to release the key from the keybed, called $\mathrm{KB}_{\mathrm{R}}$. Changes in key-bottom contact are accompanied by a large change in acceleration that coincides with the onset of pitch change. Finger-key contacts occur when the finger makes initial contact with the key surface (during depression), called $\mathrm{FK}_{\mathrm{D}}$ (Figure 1, middle panel), and when the key's spring pressure on the finger ceases during release, called $\mathrm{FK}_{\mathrm{R}}$. In contrast with $\mathrm{KBs}$, FK contacts are sometimes but not always accompanied by large acceleration changes. These kinematic landmarks may provide tactile information to the clarinetist, similar to Goebl and Palmer's (2008) findings with pianists' finger movements. Consistent with SAM, we hypothesize that increases in tactile afferent information during both key depressions and releases might facilitate the timing of an upcoming event: a keystroke that has been performed with a touch that entails a large change in acceleration (providing increased accumulation of tactile feedback) may be associated with a more accurately timed keystroke.

\section{Method \\ Participants}

Eight trained adult clarinetists ( mean $=22.2$ years old, range $=18-28$ ) with an average of 10.9 years of clarinet experience (7-20 years) and 8.5 years of instruction (420 years) participated in the study. The clarinetists were undergraduate and graduate students studying in clarinet performance at two large universities in the Montreal area. All participants regularly performed classical music, and most performed contemporary music as well. All participants reported experience performing in ensembles and playing with a metronome, being right-handed, and having normal hearing. Participants were paid a nominal fee.

\section{Stimulus Materials}

An isochronous melody was created for the experiment that would permit the independent sequential movement of each of three digits on the right hand: Index finger (Finger 2), Middle finger (Finger 3), and Ring finger (Finger 4), both in upward (release) and downward (depression) directions of motion. ${ }^{1}$ The melody was designed to be easy to perform and continuously repeated by the right hand fingers, while the left hand remained in the same position on the instrument. The melody, shown in the top panel of Figure 2, required an isolated movement of each finger: a depression of Index, Middle, then Ring finger, followed by their release in the reverse order. Thus, the melody was constructed so that only finger 2 (index finger) created an initial depression or release that could yield a Finger-Key acceleration

\footnotetext{
${ }^{1}$ Direction of motion refers to finger movement relative to the clarinet keys and not to pitch motion (or melodic contour).
} 

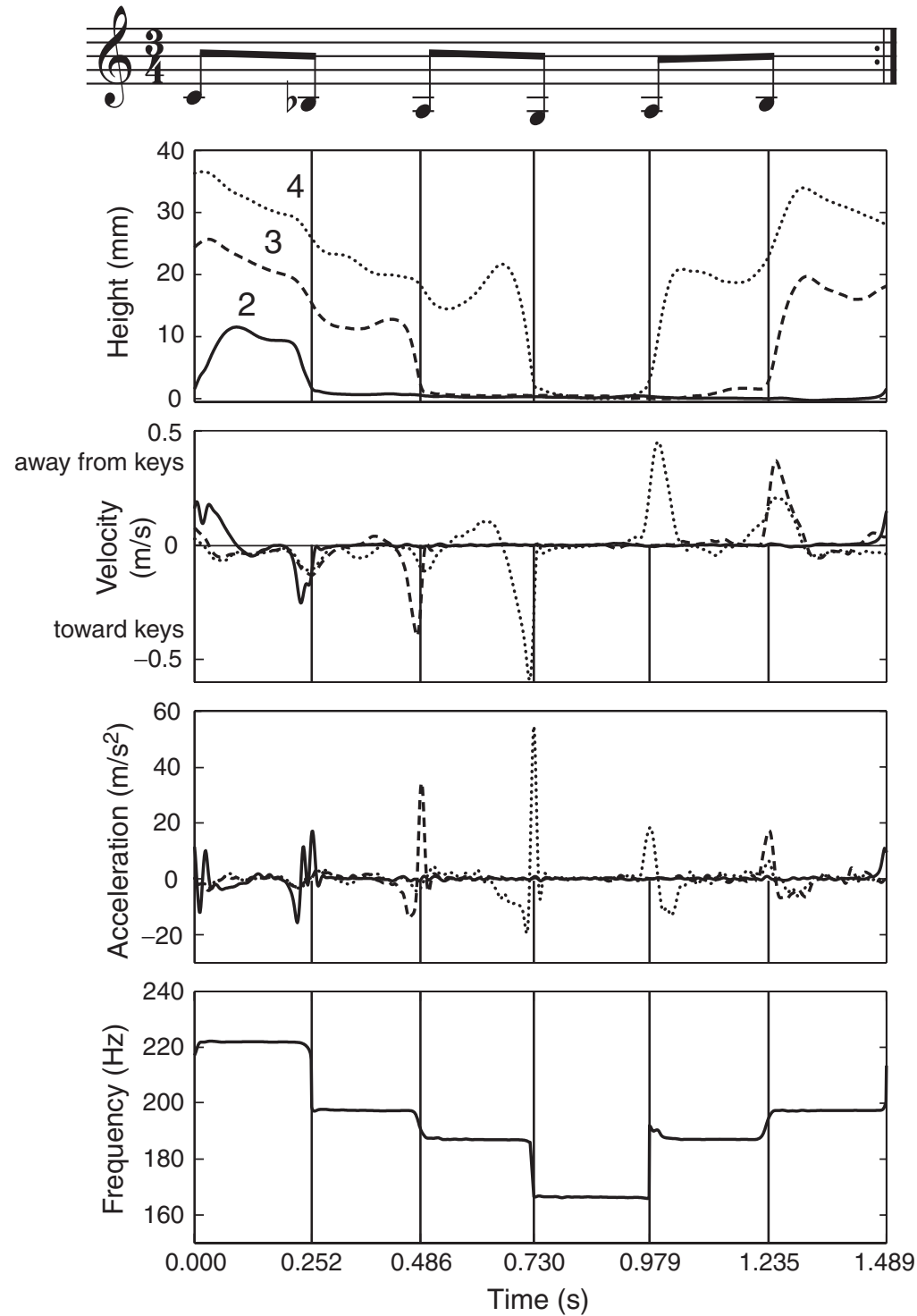

FIGURE 2. Notated melody (top panel) and finger position and acceleration trajectories (middle panels) for Fingers 2,3 , and 4, and corresponding pitch (bottom panel) from one clarinetist's performance at the medium tempo $(250 \mathrm{~ms}$ IOI). Vertical lines indicate the time of each pitch change, based on audio recording.

because of the subsequent depression/release of the linking bar. All clarinetists used the A clarinet; the notated tone C4 in Figure 2 was sounded as A3 (and so on). The melody was presented to the participants in standard musical notation with an indication to slur the tones, in a legato style.

\section{Equipment}

An Optotrak 3020 (NDI) active motion capture system was used to track the clarinetists' finger movements at a sampling rate of $167 \mathrm{~Hz}$ (approximately every $6 \mathrm{~ms}$ ).
One marker was attached to each fingertip (excluding thumbs) on each hand, and four markers were attached to the clarinet: two on the bell, one centered just above the bell, and one below the mouthpiece. Because the clarinetists moved the clarinet during performance, finger movements need to be co-registered with respect to the clarinet body position. The markers on the clarinet thus provided a reference plane against which the finger heights above the clarinet body could be measured. Each performer played on their own A clarinet; the difference in height of keys at rest and at full depression was computed $(=3 \mathrm{~mm})$ to ensure that there were not 
large differences between instruments. One instrument showed slightly lower key travel distance $(=2.5 \mathrm{~mm})$; analyses were adjusted for that instrument. A Sennheiser ME 66 standing microphone was used to record the audio at $40 \mathrm{kHz}$, and a Dr. Beat DB-88 metronome provided the synchronization signal on each quarter-note beat.

\section{Design and Procedure}

The independent variables were finger used $(2,3$, or 4$)$, direction of finger motion (key release or depression), and performance tempo $(441,250$, and $150 \mathrm{~ms}$ interonset tone intervals, or 68,120 , and $200 \mathrm{bpm}$ per quarternote beat), referred to here as "slow," "medium," and "fast" tempo conditions. Each participant performed in all conditions. Trials were blocked by tempo condition, in order from slowest to fastest. Within each trial, the melody was repeated approximately 12 times (each repetition is referred to as a "cycle"), yielding average trial durations of 55,26 , and 15 seconds in the slow, medi$\mathrm{um}$, and fast conditions respectively (after rests were removed during which participants stopped to breathe). There were two trials per tempo condition, and the first and last synchronization cycles from each trial, as well as all cycles adjacent to a rest where participants stopped to breathe, were excluded from analysis. Thus, at least 360 tones were analyzed for each participant ( 6 tones $\times 10$ cycles $\times 3$ tempi $\times 2$ trials), yielding 5352 tones in all.

Participants began the experiment by completing a questionnaire about their musical backgrounds. Data were collected for performances of two melodies, the order of which was counterbalanced across participants; the current study examines the melody that contained successive movements of individual fingers (counterbalancing order of the melodies did not affect the results reported below). Within each tempo block, participants were shown a melody and asked to memorize it. Next, the metronome was sounded and participants practiced synchronizing the melody with the metronome. Then the musical notation was removed and they performed a practice trial which was not recorded, followed by two experimental trials which were recorded. The same procedure was repeated for each melody, and then repeated for the next tempo condition. The participants were instructed to play without articulation (slurred, without breathing), and to breathe only at the end of a (6-note) cycle. They were allowed to stop playing for as long as was necessary to breathe but were asked to begin again on a metronome beat. The entire experiment lasted approximately 90 minutes.

\section{Data Analysis}

The bottom panel of Figure 2 shows the audio data from one cycle within a single participant's performance of the melody at the medium tempo $(250 \mathrm{~ms}$ interonset interval). Audio data were analyzed with Praat to extract the most prevalent pitch (the fundamental) based on autocorrelation measures applied at a sampling period of $1 \mathrm{~ms}$ (Boersma, 1993). Tone onset times were determined from audio information, which had higher temporal resolution than motion data. Steady-state frequencies were identified for each tone; tone onsets were defined by changes greater than $6.5 \mathrm{~Hz}$ from the steady-state frequency of each previous tone (corresponding to approximately half of the smallest interval distance between successive melody tones). Interonset timing (IOI, in ms) for each tone was determined by calculating the time interval from the current tone onset in the audio recording to the following one: $I O I_{x}=t_{x+1}-t_{x}$, with $x$ referring to one tone of the melody and $t_{x}$ being its pitch-based onset time.

The three-dimensional finger motion data were rotated so that the height dimension was orthogonal to the clarinet length from reed to bell, as defined by markers on the clarinet. Occasional missing data in the fingertip trajectories (less than $0.001 \%$ of all data) were interpolated. The motion data were converted to a functional form using Functional Data Analysis techniques (Ramsay \& Silverman, 2005). Order-6 b-splines were fit to the second derivative (acceleration), with knots placed on every data point, and acceleration values were smoothed using a roughness penalty on the fourth derivative $\left(\lambda=10^{-18}\right)$. The $\lambda$ parameter was chosen to generate the smallest possible generalized crossvalidation estimate compared with the raw data (Ramsay \& Silverman, 2005). Analyses were conducted on the acceleration values computed from the functional data; an example of each finger's smoothed position, velocity, and acceleration trajectories are shown in Figure 2 for one performance. Large acceleration values when clarinet keys reached bottom, indicated by vertical lines in Figure 2, accompanied each pitch change (bottom graph).

\section{Results}

\section{Timing Measures}

Clarinetists' mean interonset intervals (IOIs) were very close to prescribed metronome rates; mean fast IOI = $149.60 \mathrm{~ms}$ (prescribed $=150 \mathrm{~ms}$ ); medium IOI $=249.90$ $($ prescribed $=250)$; slow IOI $=441.90($ prescribed $=441)$, 


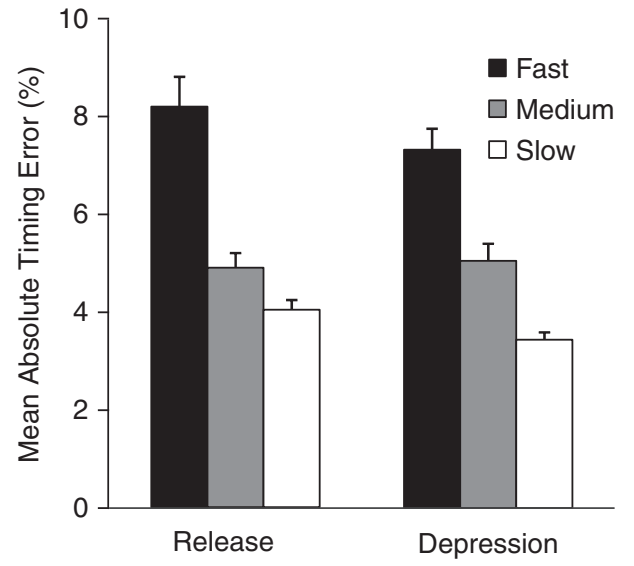

FIGURE 3. Mean absolute timing error (in percentage) by tempo condition and direction of motion.

indicating that clarinetists could successfully perform near the recommended tempi. To examine the temporal error of the IOIs, we computed the IOI error as $\left(\mathrm{IOI}_{\mathrm{obs}}-\mathrm{IOI}_{\text {exp }}\right) / \mathrm{IOI}_{\text {exp }}$, also known as "constant error" (Schmidt \& Lee, 1999). The average signed timing error (in percentage) was close to $0(M=-0.03, S E=0.38)$, as expected in conditions where a metronome provided feedback for correction.

We computed the absolute value of the timing error (called absolute error; Schmidt \& Lee, 1999) as a measure of the magnitude of temporal inaccuracy. Figure 3 shows the mean absolute error in percentage, by tempo and direction of motion. A repeated measures ANOVA by tempo (fast, medium, slow), finger $(2,3,4)$, and direction of motion (depression, release) indicated a significant main effect of tempo, $F(2,14)=104.42, p<$ .001. Posthoc comparisons confirmed that timing error increased significantly across all three tempo conditions, Tukey's HSD $=0.07, p<.05$. There were no main effects of finger or movement direction, and no interactions with tempo. There was a significant interaction between finger and movement direction, $F(2,14)=$ $7.62, p<.01$. Finger 3 showed slightly higher timing error on releases $(M=6.62 \%)$ than on depressions $(M=$ $5.12 \%)$, which may have been due to differences in finger length or mass (cf. Loehr \& Palmer, 2007). The timing error did not differ by movement direction for the other fingers (finger $2 M=5.31 \%$, finger $3 M=5.87 \%$, finger $4 M=5.37 \%$ ).

Effects of tempo on produced timing were confirmed in the variability of the IOIs. The variability of each trial was measured by the coefficient of variation $(\mathrm{CV})$, defined as $\mathrm{SD}_{\mathrm{IOI}} / \mathrm{Mean}_{\mathrm{IOI}}$. A repeated measures ANOVA on the CVs by tempo condition indicated that variability was higher at the fast tempo than at the slower tempi (mean CV at fast tempo $=.097$; medium $=.062$; slow $=$ $.047), F(2,14)=115.17, p<.001$. Posthoc comparisons confirmed that $\mathrm{CV}$ increased significantly across all three tempo conditions, Tukey's HSD $=0.012, p<.01$. Thus, both absolute timing error on a note-by-note basis and variability across each trial increased with tempo.

\section{Motion Measures}

The finger position and acceleration trajectories are shown in Figure 4 for one clarinetist's release and depression of a single tone with Finger 2. Following Goebl \& Palmer (2008), KB and FK acceleration thresholds were identified as follows: all $\mathrm{KBs}\left(\mathrm{KB}_{\mathrm{D}}\right.$ and $\left.\mathrm{KB}_{\mathrm{R}}\right)$ were identified by positive acceleration peaks exceeding $4 \mathrm{~m} / \mathrm{s}^{2}$ that occurred within $60 \mathrm{~ms}$ of the pitch change (on average, $\mathrm{KB}$ landmarks occurred $0.35 \mathrm{~ms}$ ( $\mathrm{SD}=$ $5.73 \mathrm{~ms}$ ) before the tone onsets). $\mathrm{FK}_{\mathrm{D}}$ landmarks (when
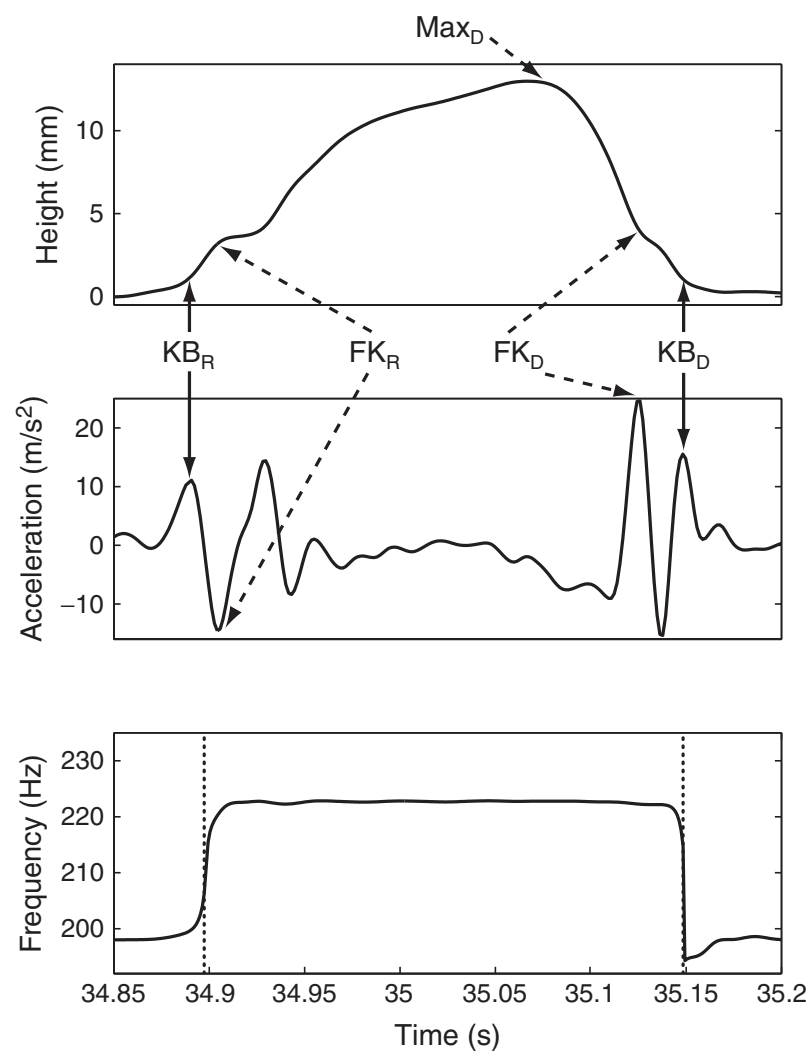

FIGURE 4. Finger 2 motion during one tone of a clarinetist's performance at the medium tempo (250 $\mathrm{ms} \mathrm{IOI).} \mathrm{The} \mathrm{finger} \mathrm{height} \mathrm{(top} \mathrm{panel)}$ and acceleration (middle panel) and corresponding pitch change (bottom panel) are shown for a key release followed by a key depression. Key-bottom (KB) landmarks are indicated with solid arrows; finger-key contact preceding key depression $\left(F K_{D}\right)$ and following key release $\left(F K_{R}\right)$ are indicated with dashed arrows. 

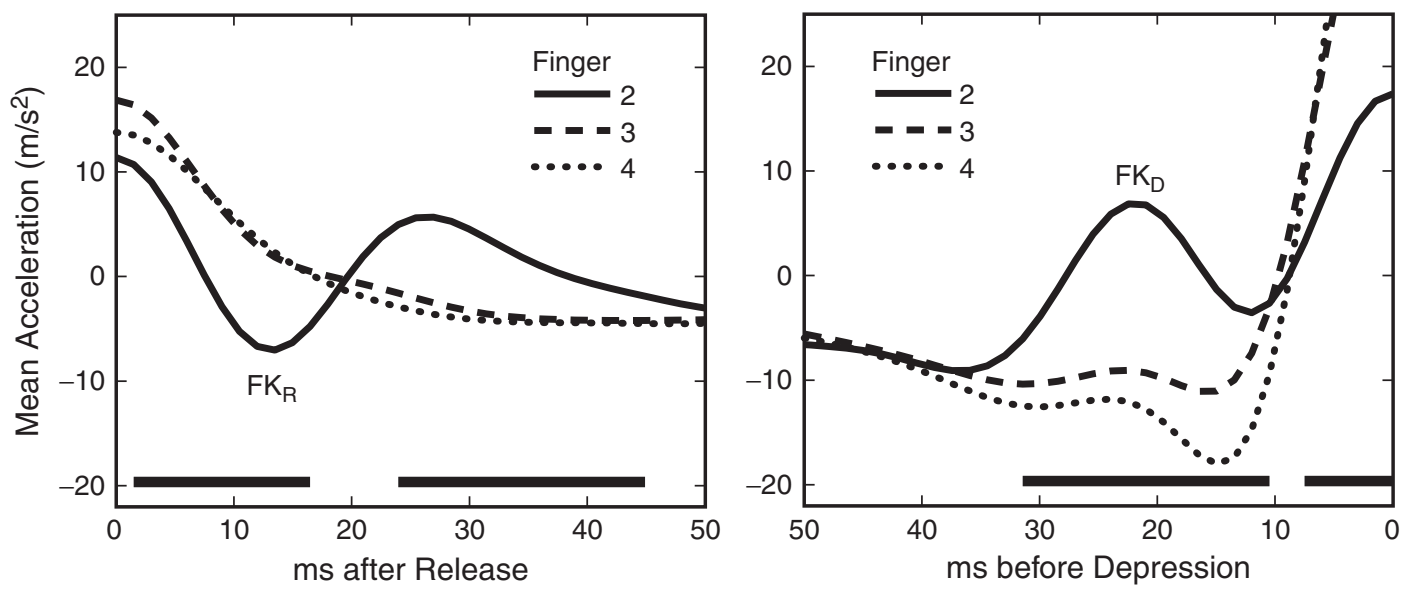

FIGURE 5. Mean finger acceleration trajectories by finger and direction of motion, after releases (left) and before depressions (right). Horizontal line at bottom indicates significance regions for finger differences at $p<.01$.

the finger has initially depressed the key from its resting position $3 \mathrm{~mm}$ above keybed) were identified when an acceleration peak greater than $4 \mathrm{~m} / \mathrm{s}^{2}$ occurred within 50 ms before the $\mathrm{KB}_{\mathrm{D}}$ landmark. $\mathrm{FK}_{\mathrm{R}}$ landmarks (when the finger releases the key at its resting position) were similarly identified when an acceleration peak (local minimum) less than $-4 \mathrm{~m} / \mathrm{s}^{2}$ occurred within $50 \mathrm{~ms}$ after the $\mathrm{KB}_{\mathrm{R}}$ landmark. ${ }^{2}$ The $+/-4 \mathrm{~m} / \mathrm{s}^{2}$ FK threshold within $50 \mathrm{~ms}$ of $\mathrm{KB}$ corresponded to the 95th percentile of all acceleration values during the event region prior to the clarinetists' keystrokes (as in Goebl \& Palmer, 2008). The identification of $\mathrm{FK}_{\mathrm{D}}$ and $\mathrm{FK}_{\mathrm{R}}$ thresholds also was constrained such that finger positions during the FK and KB landmarks were consistent with the $3 \mathrm{~mm}$ height difference between the clarinet key at rest and at key bottom, established in separate measurements. Of the total 892 depressions and releases produced by Finger 2, 79\% contained an identifiable $\mathrm{FK}_{\mathrm{R}}$ peak acceleration and $68 \%$ contained an identifiable $\mathrm{FK}_{\mathrm{D}}(100 \%$ contained an identifiable $\mathrm{KB}$, as expected). The kinematic landmarks suggest that clarinetists altered their finger dynamics in a nonlinear fashion while approaching and releasing the keys (see also Goebl \& Palmer, this issue, for similar FK-KB landmarks in piano performance).

We compared the acceleration trajectories across fingers during finger-key contact. Peak accelerations at finger-key contact were expected to be greater for Finger 2

\footnotetext{
${ }^{2} \mathrm{FK}_{\mathrm{R}}$ accelerations are negative because finger velocities upon key release are positive, indicating motion away from clarinet keys; negative $\mathrm{FK}_{R}$ accelerations indicate slowing, just as positive $\mathrm{FK}_{\mathrm{D}}$ accelerations that coincide with negative finger velocities toward clarinet keys indicate slowing.
}

than for other fingers; because the melody permitted only Finger 2's key to move upon contact, Finger 2 is the only finger motion in this melody for which an FK acceleration could occur. Figure 5 shows the mean acceleration trajectory of each finger in the $50 \mathrm{~ms}$ region following key release (left) or before depression (right). The trajectories show significant differences in acceleration across fingers, indicated by the horizontal lines at the bottom (indicating significance for $p<.01$, critical $F(2,14)=6.51)$. The significance region for releases marks a negative acceleration peak for Finger $2, \mathrm{FK}_{\mathrm{R}}$, that occurs $15.20 \mathrm{~ms}$ on average after the positive $\mathrm{KB}$ acceleration, followed by a positive acceleration as the finger departs from the key; other fingers show a gradual decrease in acceleration with no negative peak. The significance region for key depressions (right) marks a positive acceleration peak for Finger 2, $\mathrm{FK}_{\mathrm{D}}$, that occurs $21.96 \mathrm{~ms}$ on average before the positive $\mathrm{KB}$ acceleration; other fingers show little change in acceleration. Thus, the motion trajectories confirmed that only Finger 2 exhibited peak acceleration changes at finger-key contact in performances of this melody, and peak accelerations marked both key depressions and releases.

\section{Timing and Motion}

Next we address whether the peak accelerations on Finger 2's finger-key contact influenced performers' timing accuracy. Performances that contained more FK peak accelerations should exhibit greater temporal accuracy than those that did not. An ANOVA on the FK proportions in each performance by tempo and direction indicated significant differences in tempo only, $F(2,14)=$ $6.83, p<.01$, but, as shown in Figure 6, there were large 

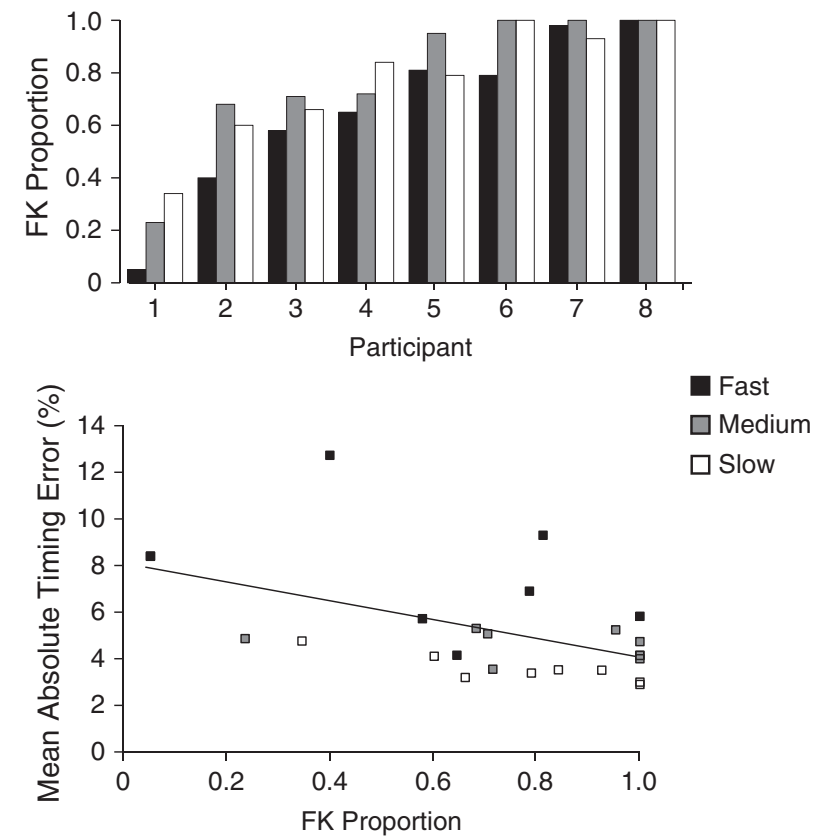

FIGURE 6. Proportion of total keystrokes containing FK landmarks by tempo condition, for clarinetists shown in increasing order (top). FK Proportion by mean absolute timing error (\%) with best-fitting regression line, by clarinetist and tempo condition (bottom).

individual differences: Some clarinetists' performances displayed high proportions of FK landmarks across all keystrokes, whereas others' did not. Figure 6 (top) shows the proportions of total keystrokes that contained FK landmarks by participant and tempo condition, listed in increasing order. To investigate whether these individual differences in finger motion at fingerkey contact corresponded to differences in temporal accuracy, as reported in piano performance (Goebl \& Palmer, 2008), we correlated each clarinetist's FK proportions for each tempo condition in Figure 6 (top) with the corresponding absolute timing error. Figure 6 (bottom) shows the significant negative correlation, $r(22)=-.50, p<.05$, between FK proportions and timing error, which indicated that those performances that contained higher proportions of peak accelerations on finger-key contact exhibited less timing error. Because faster tempo also contributed to timing error (as shown in Figure 3), a multiple regression analysis was conducted to examine relative contributions of FK proportions and produced tempo (based on the mean produced IOI for each performance) to the individual measures of timing error. The overall fit was significant, $R(21)=.75, p<.001$, and both predictors correlated negatively with timing error, FK proportion: $r(22)=-.50, p<.05$; produced tempo: $r(22)=-.68$, $p<.05$. Most important, partial correlations indicated that FK proportions predicted the timing error values above and beyond effects of produced tempo $(\beta=$ $-.34, p<.05)$. Likewise, produced tempo predicted the timing error values above and beyond FK proportions $(\beta=-.58, p<.05)$. These findings suggest that increased proportions of FK landmarks reduced timing error while faster tempo increased error in clarinet performance.

To investigate whether the FK accelerations were directly related to timing accuracy at the level of individual keystrokes, the peak finger acceleration magnitudes (absolute values) were correlated with the absolute timing error in the subsequent IOI. Both $\mathrm{FK}_{\mathrm{D}}$ and $\mathrm{FK}_{\mathrm{R}}$ landmarks represented a slowing of the finger's motion at finger-key contact; because finger motion away from keys yields a positive velocity and motion toward the keys yields a negative velocity, slowing of finger motion is indicated by negative $\mathrm{FK}_{R}$ accelerations at key release and by positive $\mathrm{FK}_{\mathrm{D}}$ accelerations at key depression. The magnitude of the FK accelerations was inversely related to the absolute timing error in the subsequent IOI at the level of individual keystrokes, both for depressions, $r(599)=-.09, p<$ .05 , and releases, $r(175)=-.18, p<.01$. In addition, timing error decreased as FK magnitude increased within the fast tempo conditions (which contained the largest timing error) for depressions, $r(175)=-.16$, $p<.05$, and releases, $r(183)=-.38, p<.01$. Thus, the individual keystroke data confirm the general pattern from the keystroke proportions: larger peak accelerations at finger-key contact are associated with greater timing accuracy.

Finally, we considered additional measures that may affect motion dynamics and temporal accuracy. We examined clarinetists' finger heights above the keys; finger height typically increases with acceleration in piano and clarinet performance. Clarinetists' maximum finger height during the IOI before depressions and following releases increased significantly with tempo, $F(2$, $14)=30.42, p<.01$; mean finger height $=21.7 \mathrm{~mm}$ in the fast tempo, $16.9 \mathrm{~mm}$ in the medium tempo, and $15.5 \mathrm{~mm}$ in the slow tempo. There were no effects of direction of motion; the different kinematic landmarks for depressions and releases were not associated with differences in finger height. We also investigated the role of individual differences in performing experience or training by correlating years of clarinet instruction and playing experience with timing accuracy measures and FK acceleration values. None of the correlations reached significance, suggesting that the relationship between timing and finger motion was consistent across the clarinetists. 


\section{Discussion}

Accurate timing is an important goal in music performance, particularly when performers must synchronize their actions. Musicians are expected to synchronize with a beat, whether that beat is generated internally or by an external source such as a metronome. We explored the role of sensory feedback from clarinetists' finger motions in controlling the timing of performance. Clarinetists' finger motion trajectories toward and away from keys contained different types and amounts of kinematic landmarks: key depressions contained peak accelerations at finger-key impact, replicating the finger-key (FK) landmarks documented in piano performance (Goebl \& Palmer, 2008). The novel finding was the change in acceleration associated with finger-key releases; peak accelerations occurred as the finger released the key, providing another instance of tactile information. Peak accelerations at finger-key contact on both key depression and release showed a positive relationship with temporal accuracy; this correlation was positive both over the entire trial and at the level of individual keystrokes. Thus, these findings are consistent with the hypothesis that tactile feedback available to performers' fingers aids the control of timing in subsequent movements during performance (Goebl \& Palmer, 2008).

Movement by the index finger generated finger-key landmarks on key depressions and releases, which were absent for other finger movements when the keys controlled by those fingers were already depressed, as predicted. This finding adds new insight to how wind instrumentalists' touch is related to the control of timing; to our knowledge, this is the first study of finger motion and synchronization in wind performance, although several have addressed the relationship of motion and timing in piano performance (Askenfelt \& Jansson, 1990; Báron, 1958; Goebl, Bresin, \& Galembo, 2005; Ortmann, 1925). The temporal accuracy of the interonset intervals increased as the magnitude of finger accelerations at finger-key contact increased, both for depressions and releases. These findings suggest that tactile feedback available at key contact aids in planning and executing upcoming events. According to the sensoryaccumulator model hypothesis (Aschersleben, 2002), multiple sensory signals that occur within a short timespan speed up the integration process; thus, tactile information associated with both finger-key contact and key-bottom contact might improve temporal accuracy in the next interval because those signals co-occur and accumulate over a short time interval (15-20 ms in the clarinet performances). This relationship suggests that sensory information from finger-key contact is important for clarinetists, even when it does not directly control intensity or pitch on wind instruments. Although individual differences were observed in the frequency with which kinematic landmarks marked the clarinetists' performances, those differences were still consistent with the general finding that timing accuracy improved in performances containing more landmarks.

There are advantages and disadvantages in applying motion capture techniques to wind instruments such as clarinets. One advantage is that we were able to measure finger motion and timing on the clarinetists' own (familiar) instruments. In contrast, most study of finger motion in piano performance is conducted on an (unfamiliar) electronic keyboard, used to avoid occlusions of fingertips that can arise with acoustic pianos (cf. Palmer, 2005). At least for key depressions, the finger dynamics were similar across clarinet and electronic piano, suggesting that the previous findings with keyboards do generalize to instruments whose finger-key contacts control timing but not sound properties such as tone intensity. A limitation of the current study is the use of simple, tightly constrained musical materials, which were designed to disentangle the complex clarinet key dynamics that result from the connecting bar between keys from the contributions of different fingers. Another constraint placed on the measurements was the sampling rate of the motion capture, which was chosen to permit simultaneous recording of the clarinet body movement separate from the finger movements, to permit their co-registration; an increase in numbers of markers used in active motion capture systems yields a decreased sampling rate. Despite these limitations and the fact that the exact frequencies and range of kinematic landmarks are specific to the mass of the clarinet keys (which are quite small compared with piano keys), the kinematic landmarks and relationship with timing replicated similar findings in piano performance (Goebl \& Palmer, 2008, 2009).

In sum, this study documents kinematic landmarks during changes in clarinetists' finger-key contact that were associated with improved timing accuracy. This association held across different performance tempi, which is known to affect timing accuracy. The fact that finger-key accelerations influence timing accuracy in clarinet and piano performance suggest that it is not instrument-specific and it is not related simply to fingersurface arrival; pitch changes in clarinet performance occur with both key depressions and releases, whereas pitch changes in piano performance occur only with key depressions. Furthermore, the consistent findings for both key releases and key depressions suggest that 
accumulation of sensory information is related to changes in sensory feedback (which is reduced during finger releases and increased during depressions), in keeping with other sensory accumulation models of perception. In addition to tactile feedback, proprioceptive information is available to musicians. For example, the clarinet's horn motion often follows a trajectory related to expressive (intentional) aspects of the performance (Wanderley, Vines, Middleton, McKay, \& Hatch, 2005), potentially providing proprioceptive feedback about the performer's body position relative to the instrument. Balasubramaniam (2006) suggested that proprioceptive feedback aids the sensory regulation of timing in the absence of any physical contact with a surface, such as moving one's fingers with an auditory pulse "in the air." How tactile feedback, proprioceptive feedback, and auditory feedback contribute to the performer's sensory regulation of timing is a rich topic for music research, for which simultaneous measurements of motion and timing in music performance are essential.

\section{Author Note}

This work was supported by the Canada Research Chairs program and NSERC Grant 298173 to the first author and by an NSERC-USRA award to the second author. We thank David Ostry and Werner Goebl for fruitful discussions, and Guillaume Houle for assistance.

Correspondence concerning this article should be addressed to Prof. Caroline Palmer, Department of Psychology, McGill University, 1205 Dr. Penfield Avenue, Montreal, QC, H3A 1B1 Canada. E-MAIL: caroline. palmer@mcgill.ca

\section{References}

Aschersleben, G. (2002). Temporal control of movements in sensorimotor synchronization. Brain and Cognition, 48, 66-79.

Aschersleben, G., Gehrke, J., \& Prinz, W. (2001). Tapping with peripheral nerve block: A role for tactile feedback in the timing of movements. Experimental Brain Research, 136, 331-339.

Aschersleben, G., Gehrke, J., \& Prinz, W. (2004). A psychophysical approach to action timing. In C. Kaernbach, E. Schröger, \& H. Müller (Eds.), Psychophysics beyond sensation: Laws and invariants (pp. 117-136). Mahwah, NJ, London: Lawrence Erlbaum.

Askenfelt, A., \& Jansson, E. V. (1990). From touch to string vibrations. In A. Askenfelt (Ed.), Five lectures on the acoustics of the piano (Vol. 64, pp. 39-57). Stockholm: Royal Swedish Academy of Music.

Balasubramaniam, R. (2006). Trajectory formation in timed repetitive movements. In M. L. Latash \& F. Lestienne (Eds.), Motor control and learning (pp. 47-54). New York: Springer.

BÁron, J. G. (1958). Physical basis of piano touch. Journal of the Acoustical Society of America, 30, 151-152.

Boersma, P. (1993). Accurate short-term analysis of the fundamental frequency and the harmonics-to-noise ratio of a sampled sound. Institute of Phonetic Sciences, 17, 97-110.

Dalla Bella, S., \& Palmer, C. (2004). Tempo and dynamics in piano performance: The role of movement amplitude. In $\mathrm{S}$. D. Lipscomb, R. Ashley, R. O. Gjerdingen, \& P. Webster (Eds.), Proceedings of the 8th International Conference on Music Perception and Cognition (pp. 256-257). Adelaide, Australia: Causal Production.

Flanagan, J. R., \& Johansson, R. S. (2002). Hand movements. In V. S. Ramashandran (Ed.), Encyclopedia of the human brain (Vol. 2, pp. 399-414). San Diego: Academic Press.
Fraisse, P. (1980). Les synchronisation sensoir-motrices aux rythmes. [The sensorimotor synchronization of rhythms]. In J. Requin (Ed.), Anticipation et comportement [Anticipation and behaviour] (pp. 233-257). Paris: Centre National de la Recherche Scientifique.

Gentilucci, M., Toni, I., Daprati, E., \& Gangitano, M. (1997). Tactile input of the hand and the control of reaching to grasp movements. Experimental Brain Research, 114, 130-137.

Goebl, W., Bresin, R., \& Galembo, A. (2005). Touch and temporal behavior of grand piano actions. Journal of the Acoustical Society of America, 118, 1154-1165.

Goebl, W., \& Palmer, C. (2008). Tactile feedback and timing accuracy in piano performance. Experimental Brain Research, 186, 471-479.

Goebl, W., \& Palmer, C. (2009). Synchronization of timing and motion among performing musicians. Music Perception, 26, 427-438.

Gordon A. M., \& Soechting, J.F. (1995). Use of tactile afferent information in sequential finger movements. Experimental Brain Resesarch, 107, 281-292.

Jones, L. (1996). Proprioception and its contribution to manual dexterity. In A. Wing, P. Haggard, \& J. R. Flanagan (Eds.), Hand and brain: The neurophysiology and psychology of hand movements (pp. 349-362). San Diego: Academic Press.

KnibestöL, M., \& VAlbo, A. B. (1980). Intensity of sensation related to activity of slowly adapting mechanoreceptive units in the human hand. Journal of Physiology, 300, 251-267.

Loehr, J. D., \& Palmer, C. (2007). Cognitive and biomechanical influences in pianists' finger tapping. Experimental Brain Research, 178, 518-528.

Macefield, V. G. (2005). Physiological characteristics of lowthreshold mechanoreceptors in joints, muscle and skin in 
human subjects. Clinical and Experimental Pharmacology and Physiology, 32, 135-144.

Ortmann, O. (1925). The physical basis of piano touch and tone. New York: E. P. Dutton \& Co.

Palmer, C. (2005). Time course of retrieval and movement preparation in music performance. Annals of the New York Academy of Sciences, 1060, 360-367.

Palmer, C., Carter, C., Koopmans, E., \& Loehr, J. D. (2007). Movement, planning, and music: Motion coordinates of skilled performance. In E. Schubert, K. Buckley, R. Elliott, B. Koboroff, J. Chen, \& C. Stevens (Eds.), Proceedings of the International Conference on Music Communication Science (pp. 119-122). Sydney, Australia: University of Western Sydney.
Ramsay, J. O., \& Silverman, B. W. (2005). Functional data analysis (2nd ed.). New York: Springer.

Schmidt, R. A., \& LeE, T. D. (1999). Motor control and learning: A behavioral emphasis (3rd ed.). Champaign, IL: Human Kinetics.

Wanderley, M. M., Vines, B., Middleton, N., McKay, C., \& HATCH, W. (2005). The musical significance of clarinetists' ancillary gestures: An exploration of the field. Journal of New Music Research, 34, 97-113.

Werner, G., \& Mountcastle, V. B. (1965). Neural activity in mechanoreceptive cutaneous afferents: Stimulus-response relations, Weber functions, and information transmission. Journal of Neurophysiology, 28, 359-397. 
